\section{De la traversée du désert au prix Nobel de Physiologie ou Médecine décerné à James Allison et Tasuku Honjo : la longue marche de l'immunothérapie des cancers}

\section{Entretien avec Wolf Hervé Fridman}

Wolf Hervé Fridman ${ }^{1}$, Jean-Luc Teillaud ${ }^{2}$

Jean-Luc Teillaud: Pour l'immunologiste et l'immunothérapeute des cancers que tu es, ce prix Nobel de Physiologie ou Médecine décerné à James Allison et Tasuku Honjo, c'est une grande victoire, une grande reconnaissance pour les scientifiques et cliniciens de ce champ, non?

Wolf Hervé Fridman : Oui, pour le champ de l'immunologie du cancer mais aussi de l'immunothérapie ! C'est évidemment une reconnaissance qui fait du bien parce que c'est un champ qui a été ignoré et méprisé pendant très longtemps. Cela fait cinquante ans que je suis dans la discipline et nous sommes passés par des périodes de traversée du désert extrêmement longues, où, malgré les éléments certes souvent indirects mais convergents - le fait que les cellules du système immunitaire étaient capables de reconnaître leurs propres cellules cancéreuses, que la stimulation du système immunitaire, en tout cas dans des modèles expérimentaux, pouvait induire des régressions tumorales, que les individus qui avaient des déficits immunitaires étaient beaucoup plus enclins à développer des cancers, que des sujets qui avaient eu des transplantations et étaient traités par immunosuppresseurs, développaient souvent des lymphomes - malgré tous ces éléments accumulés pendant des années, la communauté scientifique et médicale était goguenarde voire agressive, rejetant l'idée d'un contrôle des cancers par le système immunitaire! Le fait que celui-ci soit désormais reconnu comme un acteur majeur de la défense contre le cancer et que sa stimulation et sa modulation puisse induire des réponses thérapeutiques capables de contrôler la maladie cancéreuse pendant des années, est une grande victoire et un immense succès.

Entretien réalisé le 16 octobre 2018 par Jean-Luc Teillaud. Vignette (Photos III. Niklas Elmehed. ( ) Nobel Media).

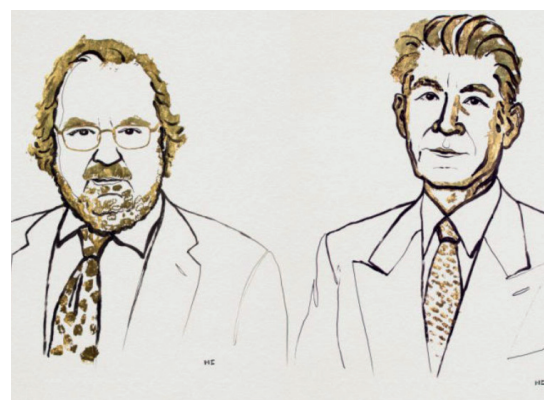

${ }^{1}$ Professeur émérite à I'université Paris-Descartes, président du cancéropôle Île-de-France, ancien président du conseil scientifique de l'association pour la recherche sur le cancer et ancien directeur du Centre de Recherche des Cordeliers et de l'unité Inserm 255.

${ }^{2}$ Rédacteur en chef de médecine/sciences, Équipe «Microenvironnement immunitaire et immunothérapie », Centre d'Immunologie et des Maladies Infectieuses (CIMI) - Inserm UMRS 1135 - Sorbonne Université, 91, boulevard de l'Hôpital, 75013 Paris, France.

jean-luc.teillaud@inserm.fr

L'établissement initial d'une relation entre cancer et immunité n'a pas été une découverte fondamentale. C'est celle d'un chirurgien new-yorkais, William Coley, à la fin du XIXe siècle, qui a observé la régression d'un sarcome chez un de ses patients cancéreux ayant développé une infection aiguë [1]. Cela a été à l'origine de la toxine de Coley ${ }^{1}$ et du traitement de patients avec des extraits bactériens. Cette observation initiale a conduit à un lointain héritage encore présent aujourd'hui : le premier, c'est la découverte en 1975 du tumor necrosis factor (TNF ou facteur de nécrose tumorale) dans le sérum de souris auxquelles avaient été injectés des extraits bactériens à des fins anti-tumorales [2] ; le second, c'est I'utilisation du BCG (Bacillus Calmette-Guérin) comme traitement du cancer superficiel récidivant de la vessie [3]. Malgré ces découvertes, II y a eu une très longue traversée du désert

${ }^{1}$ La toxine de Coley était un mélange un mélange de bactéries Serratia marcescens et de Streptococcus pyogenes inactivées par la chaleur [46]. 
pendant laquelle cette relation entre système immunitaire et contrôle des tumeurs n'a pu être réellement démontrée pour différentes raisons : les modèles animaux, en particulier murins, avaient été essentiellement explorés avant la découverte des molécules de l'histocompatibilité ; c'étaient des greffes de tumeurs allogéniques ${ }^{2}$ qui étaient bien entendu rejetées ; lorsque les greffes sont devenues syngéniques ${ }^{2}$, on a pu poser des questions sur l'immunité de transplantation plus que sur la reconnaissance immunitaire de cancers spontanés. La démonstration du rôle de l'immunité dans le contrôle anti-tumoral, bien que suggéré dès 1957 par Sir Frank Macfarlane Burnet [4], a été vraiment longue à être apportée.

JLT : Si on avance dans le temps, quelles sont les données qui ont vraiment fait faire un bond en avant et ont conduit, y compris des oncologues célèbres - je pense à Robert Weinberg ${ }^{3}$ - à reconnaître que c'était la fin de la traversée du désert, le début d'une nouvelle histoire pour l'immunologie des cancers? Quels ont été les éléments et là, j'en viens à nos deux récipiendaires, est-ce que ceux-ci ont été à l'origine de ces premières fractures de fin de traversée du désert ou est-ce qu'ils n'interviennent que plus tardivement?

WHF : Je citerais des éléments de recherche fondamentale et des éléments de recherche thérapeutique. Pour la recherche fondamentale, il y a trois découvertes qui ont à mon sens joué un rôle majeur.

1. La première découverte, c'est la démonstration par Thierry Boon (Ludwig Institute for Cancer Research, Bruxelles, Belgique) que des lymphocytes $T$ de malades atteints de mélanome reconnaissent des peptides présentés par les cellules tumorales, des peptides du soi mais d'un soi particulier, car dérivés d'antigènes carcino-embryonnaires, appelés maintenant antigènes « cancer testis » [5].

2. La seconde découverte, qui me semble être très déterminante, c'est celle de Schreiber (Department of Pathology and Immunology, Washington University School of Medicine, St. Louis, États-Unis) [6] : elle repose sur une observation et sur l'élaboration d'une théorie, celle des $3 \varepsilon$, à partir d'expériences menées chez la souris.

- Il montre que $100 \%$ des souris sans système immunitaire, ni adaptatif, ni inné, développent des tumeurs, dont $80 \%$ sont des tumeurs malignes.

- Il décrit trois étapes majeures du développement tumoral. La première, c'est ce qu'il appelle le premier $\varepsilon$ (pour elimination) : quand il n'y a pas de cellules immunitaires, on n'élimine pas les cellules cancéreuses, ce qui a contrario revient à dire que s'il y en a, on peut les éliminer! Cela, c'est une découverte en creux, d'une certaine façon. La seconde étape, essentielle, car, à mon avis la plus pertinente pour la prise en charge clinique, c'est la phase d'équilibre (le second $\varepsilon$ pour

\footnotetext{
${ }^{2}$ Une greffe allogénique est une greffe de tissus entre individus non apparentés qui diffèrent par leurs molécules d'histocompatibilité, différences qui conduiront au rejet de la greffe. A contrario, une greffe est dite syngénique lorsqu'elle est faite entre individus qui ont des molécules d'histocompatibilité identiques, comme dans le cas de souches de souris consanguines ou de vrais jumeaux chez l'homme.

${ }^{3}$ Robert Weinberg est un célèbre chercheur en oncologie moléculaire au Whitehead Institute for Biomedical Research (MIT, Massachussets Institute of Technology, Cambridge, États-Unis) qui, outre des articles majeurs dans ce domaine, a écrit de remarquables synthèses sur l'oncologie mais où l'immunité antitumorale était totalement absente. En 2011, il a publié un manuscrit [47] qui fait désormais date où il a adoubé l'irruption de l'immunité anti-tumorale dans la recherche sur le cancer en utilisant une figure très similaire à celle qu'il avait utilisé 10 ans auparavant mais qui ne soufflait alors mot de l'immunité anti-tumorale!
}

equilibrium). La tumeur commence à se développer mais le système immunitaire continue à être efficace, soit en tuant les cellules tumorales, soit en les maintenant dans un état dormant, sans que les mécanismes mis en jeu soient très clairs, même s'il est désormais bien établi que des cytokines comme l'interféron, en particulier l'interféron gamma (IFN- $\gamma$ ) jouent un rôle important. Cette phase d'équilibre peut durer des années et être levée en bloquant I'IFN- $\gamma$ par exemple. Quand un cancer devient cliniquement détectable, il est déjà au moins dans cette phase-là. Enfin, la troisième étape, c'est l'échappement des cellules tumorales à la surveillance par le système immunitaire (le troisième $\varepsilon$ pour escape), en mutant et/ou en se débarrassant des molécules reconnues par celui-ci si elles ne leur sont pas indispensables.

Tout cela, c'est le corpus intellectuel, théorique, élaboré par Robert Schreiber au début des années 2000 [6], qui a permis la suite.

3. La troisième découverte, c'est celle faite par Jérôme Galon et Franck Pagès dans mon laboratoire du Centre de Recherche des Cordeliers (CRC) (Inserm UMRS 1138, Paris) en 2005-2006 [7]. Nous avons été en effet les premiers à montrer que l'infiltrat immunitaire, en particulier les lymphocytes T mémoires, sont le plus fort facteur de prédiction de l'évolution d'un patient cancéreux. Cette démonstration a vraiment changé le paradigme et a conduit à l'idée qu'il était essentiel d'étudier le microenvironnement immunitaire tumoral pour comprendre le développement des tumeurs. L'analyse de ce microenvironnement et la découverte, également dans mon laboratoire, par Marie-Caroline Dieu-Nosjean, de la présence de structures lymphoïdes tertiaires au sein des tumeurs [8], véritables lieux de production de réponses antitumorales, dont l'existence était alors seulement connue dans des pathologies infectieuses et auto-immunes, nous ont amenés à considérer le microenvironnement tumoral comme un organe immunitaire à part entière. Nous pouvons dire que, d'une certaine façon, le cancer est une maladie immunologique et que les tumeurs sont des lieux au sein desquelles la réaction immunitaire joue un rôle majeur pour leur destin.

JLT : Cela, c'est sur le plan fondamental, mais sur le plan clinique?

WHF : sur le plan clinique, il y a quelques événements à retenir. Le premier bond en avant fût la greffe de moelle osseuse allogénique pour traiter des leucémies aiguës; le principe n'était pas un principe immunologique : l'idée était de traiter le patient afin d'éliminer ses cellules leucémiques pour les remplacer par les cellules saines. Le premier patient qui a été traité le fût par une exsanguino-transfusion. C'est ce qui a fait la gloire de Jean Bernard (alors à l'hôpital Hérold à Paris avant de 
fonder l'Institut de Recherche sur les Maladies du Sang à l'hôpital Saint-Louis) en 1947 [9] : ce patient a eu une rémission grâce au sang transfusé ; les décennies suivantes ont montré que les résultats étaient meilleurs lorsque c'était de la moelle osseuse qui était greffée, puis les cellules souches hématopoïétiques du sang ont été utilisées avec succès. Là, on est au début des années 1960, 1959 la première greffe réussie par Georges Mathé (alors Assistant de Jean Bernard à l'hôpital Saint-Louis et qui sera le fondateur de l'Institut d'hématologie et de cancérologie de l'hôpital Paul-Brousse à Villejuif) [10] ! On obtient deux effets : l'effet GVH (graft versus host, réaction du greffon contre l'hôte), des cellules de la moelle allogénique s'attaquent aux cellules du receveur du fait de l'allo-réactivité ; l'effet GVL (graft versus leukemia, réaction du greffon contre les cellules leucémiques), qui résulte en une destruction des cellules leucémiques du patient par les lymphocytes T du donneur. Cela a été le premier effet immunologique observé en clinique, même si l'air du temps était de dire que l'immunothérapie ne marchait pas, ce qui était de toute évidence faux!

Le second bond en avant, ce sont les travaux de Steve Rosenberg au NCl (Surgery Branch, National Cancer Institute, Bethesda, États-Unis), qui traite des patients cancéreux incurables qui ont des mélanomes ou des cancers des reins métastatiques, pour lesquels la chimiothérapie n'a plus aucun effet, avec de l'interleukine 2 (IL-2). Le papier de Rosenberg, publié en 1985 [11], montre des régressions spectaculaires chez certains des patients traités. Malgré beaucoup de toxicité, une AMM (autorisation de mise sur le marché) a été délivrée pour l'IL-2 dans les mélanomes et les cancers du rein métastatiques. Quelques mois plus tard, Rosenberg publie un autre papier où, pour la première fois, il utilise des cellules provenant du sang périphérique, activées in vitro, des LAK (lymphokine-activated killers), probablement des cellules NK (natural killer) activées, en combinaison avec l'IL-2 [12]. Ce travail pionnier a été salué par un éditorial du New England Journal of Medicine «Immunotherapy of cancer, the end of the beginning ? ». En effet, ce n'était pas le début de la fin mais bien la fin du début. En 1988, il publie I'utilisation de TIL (tumor infiltrating lymphocytes) dans le mélanome avec un taux de réponse très impressionnant [13] : ce sont ces lymphocytes qui infiltrent la tumeur qui sont utilisés dans ces débuts balbutiants de l'immunothérapie! Ces travaux assoient l'immunothérapie moderne. L'autre grande date, c'est l'ASCO 2010 (American Society of Clinical Oncology): la régression de tumeurs par l'anticorps anti-CTLA-4 (cytotoxic T lymphocyte activated-4) y est rapportée [14]. Quelques mois plus tard, c'est au tour de l'anticorps anti-PDl (programmed death-1), qui fait également régresser des tumeurs [15]. Un point remarquable : ces deux molécules ont cassé les règles de l'enregistrement des médicaments, car elles ont été enregistrées à la suite d'essais de phase 1 tellement les résultats étaient impressionnants!

JLT : Et de plus, pour les anti-PD-1, sans une indication de cancers particuliers?

WHF : oui, on s'adresse directement au système immunitaire de l'hôte, en faisant l'hypothèse qu'il sera capable de contrôler la tumeur, quel que soit son type. Pour être complet, il faut aussi parler du travail qui représente un chemin de traverse par rapport aux TIL, l'élaboration et l'utilisation des CAR T (chimeric antigen receptor $T$ cell) qui, sur la base du premier travail de Zelig Eshhar au Department of Chemical Immunology, Weizmann Institute of Science, à Rehovot (Israël) en 1989 [16], et grâce aux travaux de Carl June au Center for Cellular Immunotherapies (Perelman School of Medicine, University of Pennsylvania, Philadelphie, États-Unis) [17] mais d'autres aussi, Michel Sadelain au Memorial Sloan-Kettering Cancer Center (MSKCC) à New York (États-Unis) [18] et Phil Greenberg, au Fred Hutchinson Cancer Research Center à Seattle (États-Unis) [19] ont ...

JLT : mélangé la thérapie cellulaire et les anticorps

WHF : oui, en faisant exprimer par exemple par des lymphocytes $T$ des fragments d'anticorps dirigés contre des antigènes associés aux tumeurs, fusionnés à des séquences intracellulaires d'activation des lymphocytes $T$.

JLT : dans les hémopathies malignes

WHF : dans les hémopathies malignes ; cela a conduit à cet extraordinaire nouveau modèle économique mis en place par Novartis, qui est celui du «satisfait ou remboursé ». C'est cher, mais si cela marche.

Parallèlement ou un peu avant, il y a eu l'utilisation des anticorps monoclonaux anti-tumeur, initiée par Ronald Lévy (Department of Medicine, Division of Oncology, Stanford Cancer Institute, Stanford University, Stanford, États-Unis) avec un anticorps anti-idiotype pour traiter le lymphome $B$ [20]. En oncologie, le premier grand succès a été l'anticorps anti-CD20, le rituximab, qui a révolutionné la prise en charge du lymphome. Pendant longtemps, on a considéré que l'on faisait, avec ces anticorps, de la chimiothérapie avec de grosses molécules à effet cytotoxique immédiat. II a fallu attendre assez récemment pour que des travaux faits par Fu [21] (Department of Pathology and Committee on Immunology, University of Chicago, Chicago, États-Unis) et à Paris par toi-même (au CRC) [22], montrent que, pour qu'il y ait un effet antitumoral à long terme, il faut la mise en place d'une mémoire immunitaire. Cela soutient le concept que toute approche thérapeutique en oncologie, pour qu'elle soit efficace à long terme, nécessite l'activation du système immunitaire, et est de facto une immunothérapie, comme I'ont montré par exemple Guido Kroemer (CRC) et Laurence Zitvogel (Institut de Cancérologie Gustave Roussy Cancer Campus [GRCC], Unité Inserm 1015, Villejuif) pour la radiothérapie et la chimiothérapie [23]. Mais venons-en maintenant à la découverte qui a valu le prix Nobel en octobre 2018 à James Allison et Tasuku Honjo.

JLT : en effet, revenons à ces deux chercheurs : des personnages extrêmement différents, dans leurs parcours également?

WHF : I'histoire de ce prix Nobel est une histoire très intéressante. Le premier élément, essentiel, c'est le fait que tous les deux sont des fon- 
damentalistes et font une découverte de fondamentalistes. Commençons par Tasuku Honjo (Immunology and Genomic Medicine, Graduate School of Medicine, University of Kyoto, Japon) parce qu'il aurait pu déjà avoir ce prix avant sa découverte de PD-1 ! Honjo, c'est une filiation : c'est l'homme qui, à la suite des travaux de Susumu Tonegawa qui a reçu le prix en 1987 pour ses travaux menés à l'institut d'immunologie de Bâle (Suisse) [24], et suivant en cela tous les deux les travaux de Pierre Chambon (Institut de Génétique et de Biologie Moléculaire et Cellulaire, IGBMC, IIlkirch) et Phil Leder (alors au Laboratory for Molecular Genetics aux National Institutes of Health, NIH, Bethesda, États-Unis) montrant qu'il y a des introns et des exons dans les gènes eucaryotes [25, 26], va tout d'abord s'intéresser aux événements moléculaires contrôlant le réarrangement des gènes d'immunoglobuline. Tonegawa a montré que la formation d'une région variable est due à un réarrangement de gènes codant ses différentes parties, ce qui permet l'obtention d'un vaste répertoire [24]. Honjo, lui, analyse le mécanisme moléculaire par lequel se fait la commutation de classe, c'est-à-dire la transformation d'une IgM (immunoglobuline M), l'anticorps produit lors d'une réponse primaire, en un anticorps de plus forte affinité lors de la réponse secondaire, qui peut être une $\operatorname{lgG}$, une $\lg A$, une $\lg \varepsilon$... II montre que cette commutation est due à un mécanisme d'excision d'un morceau d'ADN, permettant d'associer le gène réarrangé du domaine variable à un nouveau gène codant la chaîne lourde d'IgG ou d'IgA, ou d'Igદ [27-29]. Il va d'ailleurs en parallèle découvrir une enzyme, AID (activationinduced cytidine deaminase) [30], qu'il montre, en même temps qu'Anne Durandy (Inserm U429, hôpital Necker, Paris) [31], être indispensable à la commutation de classe [32] ainsi qu'au mécanisme d'hypermutation somatique qui cible les domaines variables des immunoglobulines, conduisant à la formation d'anticorps de haute affinité [32]. Pour ces découvertes exceptionnelles, il aurait pu avoir le prix Nobel. Mais il ne l'a pas eu Tasuku Honjo continue ses recherches en analysant comment se fait la sélection des thymocytes dans le thymus, entre les $95 \%$ qui vont mourir et ceux qui deviennent des lymphocytes circulants ou présents dans les organes lymphoïdes, assurant l'intégrité du soi et la lutte contre les infections et les cancers ; il décrit alors en 1992 une molécule, qu'il appelle programmed cell death 1, PCD-1 (qui deviendra programmed death-1, PD-1, CD279), impliquée dans le choix entre la mort et la survie des thymocytes [33]. Au cours des années suivantes, entre 1994 et 1999, il montre le rôle majeur que PD-1 peut jouer dans des modèles de maladies auto-immunes par son rôle inhibiteur de l'activation T [34-36] et il décrypte en 2001 le mécanisme moléculaire par lequel l'engagement de PD-1 conduit à l'inhibition du lymphocyte T [37]. J'en profite pour rappeler qu'il y a une autre découverte reliée faite en 1992 dans mon laboratoire par Sebastian Amigorena et Christian Bonnerot, en partie en collaboration avec Ira Mellman (qui était alors au Department of Cell Biology, Yale University School of Medicine, New Haven, et désormais chez Genentech, South San Francisco) [38], qui est celle du motif appelé ITIM (immunoreceptor tyrosine inhibition motif), que nous avons décrit les premiers sur les récepteurs $\mathrm{Fc}^{4}$ des IgG inhibiteurs de plusieurs cellules, notamment des lymphocytes $B$, motif présent sur beaucoup

${ }^{4}$ Récepteur de la région $\mathrm{Fc}$ des immunoglobulines $\mathrm{G}(\lg \mathrm{G})$. de récepteurs régulateurs, en particulier sur ceux dont nous parlons aujourd'hui [37]. C'est l'un des mécanismes majeurs par lequel passe l'inhibition induite dans les lymphocytes T par PD-1 et CTLA-4.

Dès 2002, Tasuku Honjo et son équipe montrent une inhibition de la tumorigenèse chez des souris dont le gène codant PD-1 a été invalidé [39], puis publie des articles montrant qu'un anticorps anti-PD-LI (I'un des ligands de PD-1 exprimé sur les cellules tumorales) bloque également la tumorigenèse et qu'un anticorps anti-PD-1 inhibe l'apparition de métastases dans un modèle murin de mélanome [40]. D'autres chercheurs ont accompagné cette découverte, Lieping Chen, Gordon Freeman, et Arlene Sharpe, le premier à l'université de Yale à New Haven, les deux autres à l'université de Harvard à Boston, et vont contribuer à démontrer que le blocage, grâce à des anticorps anti-PD-Ll et antiPD-1, de l'interaction entre PD-1 et PD-LI permet d'avoir un effet anti-tumoral.

James Allison est un personnage tout à fait différent. Jim Allison (il n'utilise pas son «vrai » prénom, James), qui est depuis 2012 au MD Anderson Cancer Center de Houston, c'est un Texan d'origine, qui a mené ses recherches pionnières au Cancer Research Laboratory de Berkeley et qui a été ensuite de 2004 à 2012 à New York (au MSKCC), par ailleurs excellent guitariste et joueur de jazz, qui a créé un groupe qui s'appelait «The checkpoints », et, je crois maintenant, les «Checkmates».

JLT : qui avait d'ailleurs quitté son collège où l'on ne voulait pas enseigner la théorie darwinienne de l'évolution; il s'en est enfui !

WHF : oui, un phénotype très différent, mais lui aussi un fondamentaliste. Allison, sa passion, c'était le TcR (récepteur T de l'antigène) et comprendre comment un lymphocyte $T$ s'active. Il est d'ailleurs parmi les premiers à comprendre que l'engagement du TcR ne suffit pas pour cette activation et qu'il faut des molécules accessoires [41]. Parmi celles-ci, il y a CD28, rendue tristement célèbre lors d'un essai thérapeutique ${ }^{5}$, qui reconnaît une molécule appelée à l'époque $B 7$, main-

\footnotetext{
${ }^{5}$ Cet essai de phase 1 fût mené à Londres en 2006 chez six volontaires sains qui reçurent une dose unique d'un anticorps anti-CD28, développé par une firme allemande, TGenero, pourtant définie lors d'études précliniques menées chez des primates non-humains, qui provoqua une très violente tempête cytokinique (caractérisée par une production massive de cytokines pro-inflammatoires) suite à l'activation massive des lymphocytes $T$ de ces sujets, dont quatre souffrirent de défaillance multisystémique (ou syndrome de défaillance multiviscérale, SDMV). II n'y eu heureusement aucun décès mais ce grave incident amena la FDA (Food and Drug Administration) américaine et l'EMEA (European Medicines Agency) à émettre de nouvelles règles beaucoup plus strictes que les précédentes quant aux doses utilisées et à l'escalade de doses effectuée lors des essais cliniques de Phase 1 (le NOAEL, « No Observable Adverse Effect Level », pour la toxicologie et le MABEL, «Minimal Anticipated Biological Effect Level » pour la pharmacologie, afin de déterminer la dose initiale maximale recommandée de départ).
} 
tenant CD80 (B7.1) et CD86 (B7.2) à la surface des cellules dendritiques. Allison montre qu'il est important qu'il y ait une interaction entre CD28 et B7 afin d'induire une activation de plus longue durée dans le lymphocyte $T$, de façon concomitante à la reconnaissance des complexes peptidiques par le TcR. Parallèlement, il réalise qu'une autre molécule, CTLA-4, a le même ligand que CD28. II montre alors, comme d'autres, notamment Jeffrey Bluestone (au Ben May Institute and the Committee of Immunology, The University of Chicago et actuellement directeur du Sean N. Parker Autoimmune Research Laboratory, University of California, San Francisco, États-Unis) [42], que CTLA-4 bloque l'activation des lymphocytes T [41]. L'expression de CTLA-4 est induite par l'activation du lymphocyte $T$ et entre en compétition pour le même ligand avec CD28, freinant ainsi l'hyper-activation à laquelle l'activation T peut conduire. Tout comme Bluestone, il fait l'hypothèse que CTLA-4 joue un rôle majeur dans des maladies autoimmunes [41, 42]. Les personnes dépourvues de CTLA-4 développent une maladie auto-immune multi-organes extrêmement invalidante.

Pourquoi cette molécule s'appelle-t-elle CTLA-4 ? C'est la découverte d'un Français, au Centre d'Immunologie de Marseille-Luminy (CIML), Pierre Golstein. Pierre a passé une grande partie de sa carrière à travailler sur les lymphocytes tueurs, les cytotoxic $T$ lymphocytes (CTL). Au milieu des années 1980, il construit une banque différentielle d'ADNc (ADN complémentaire), pour définir des molécules exprimées par un lymphocyte Tactivé («CTLA ») et pas par un lymphocyte non activé. $\varepsilon t$ il trouve un certain nombre de gènes qu'il appelle CTLA-1 (pour « cytotoxic T-lymphocyte-associated antigen $1 \gg), A-2, A-3, A-4, A-5$, etc. [43].

JLT : un peu comme les OKTI, 2, 34 de Pei Cheng Kung ${ }^{6} .$. WHF : oui, ou les Dal, 2, 3, 4... de Jean Dausset ${ }^{7}$ avant que cela ne devienne HLA (human leukocyte antigen); parmi les CTLA, il y en a au moins quatre qui sont devenus célèbres: CTLA-1, c'est le granzyme $B, C T L A-3$, le granzyme A, CTLA-8, l'IL-17... et CTLA-4 ! CTLA-4 a été longtemps connu dans la littérature sans que l'on ne soupçonne son implication dans le cancer. Qu'est-ce qui fait que c'est Jim Allison qui teste un anticorps antiCTLA-4 dans un modèle murin anti-tumoral ? En fait,

\footnotetext{
${ }^{6}$ Pei Cheng Kung était un chercheur de l'université de Harvard qui a généré en 1977 une série d'anticorps monoclonaux dirigés contre les lymphocytes Thumains, qu'il a désignés sous le nom d'OKT- $1,-2,-3$ (l'antigène reconnu est devenu CD3), - 4 (CD4), $-5,-6,-7,-8$ (CD8), etc. 0 pour Ortho (firme qui avait financé l'étude), K pour Kung et T pour «T-cell».

${ }^{7}$ Jean Dausset, prix Nobel de Physiologie ou Médecine en 1980 avec Baruj Benacerraf et Georges Snell pour leur découverte des molécules de l'histocompatibilité chez l'homme et la souris, avait en effet initialement appelé les antigènes qu'il avait découverts Da-1, $-2,-3$, etc. (pour Dausset-1, Dausset-2, ...).
}

c'est ce qu'il m'a raconté alors que nous participions tous les deux au même meeting à New York, ses deux parents sont morts d'un cancer, plusieurs autres membres de sa famille sont morts d'un cancer, et ce fondamentaliste a décidé alors de regarder dans un modèle murin tumoral si un anticorps anti-CTLA-4 avait un effet ! Et la réponse a été positive! II injecte seulement l'anticorps et la tumeur régresse [44]. Alors, et c'est la force de Jim Allison, il se dit « je veux en faire un médicament, je veux aller jusqu'au bout ». Tout en continuant ses recherches fondamentales [45], il réussit à intéresser un collègue et ami, Alan Korman (de la firme américaine Medarex, Annandale, New Jersey, États-Unis), qui produit un anticorps humain dans des souris humanisées, appelé ipilimumab, capable de lier et d'inactiver CTLA-4. Finalement, le premier essai de phase 3 publié en 2010 [14] montre des résultats spectaculaires chez des patients atteints de mélanome métastatique, ce qui conduit la FDA (food and drug administration) à autoriser l'utilisation de l'ipilimumab chez des patients présentant des mélanomes métastatiques. Et tout ce travail

JLT : a donné naissance à toute une génération d'anticorps antagonistes dirigés contre différentes molécules inhibitrices de la réponse $T$, et qui ont été appelés inhibiteurs de points de contrôle (anti-immune checkpoints, ICP).

WHF : oui, ces travaux ont consisté à porter ces découvertes jusqu'à l'outil thérapeutique et font que ce prix Nobel est tout à fait justifié. D'abord, ce sont des histoires individuelles formidables, de fondamentalistes. Cela montre bien que la recherche fondamentale, c'est essentiel. Jim, dans le premier discours qu'il a tenu, quand on lui a dit qu'il allait avoir le prix Nobel, dans ce congrès à New York, a dit que, s'il avait travaillé sur la thérapeutique du cancer, il n'aurait jamais découvert une molécule thérapeutique de cette qualité-là. Parce qu'il aurait essayé d'améliorer des molécules, et, de la même façon que l'on ne découvre pas l'électricité en améliorant la chandelle, c'est grâce à la recherche fondamentale qu'il a pu avancer. Ce sont César Milstein ${ }^{8}$ et Georges Köhler ${ }^{8}$ qui découvrent les anticorps monoclonaux au travers de leurs recherches sur la structure et la spécificité des anticorps [48], ce sont Pierre Golstein, Tasuku Honjo, Jim Allison, Robert Schreiber et tous les autres chercheurs qui font des découvertes qui vont véritablement à terme changer la vie des patients.

Nous sommes au début d'une nouvelle histoire de la cancérologie ; on ne traite plus la cellule cancéreuse, on traite le patient qui, dans la grande majorité des cas, a su contrôler son cancer pendant des années; on va donc lui permettre de continuer à le contrôler. Bien sûr, il y a encore beaucoup de patients qui ne répondent pas, bien sûr, les mécanismes fins ne sont pas connus complètement, bien sûr, il y a des résistances primaires et secondaires, par modulation des molécules d'histocompatibilité etc. qui se mettent en place, mais ce changement de paradigme est historique. Ce sont moins les résultats cliniques à ce jour que ce changement de paradigme que marque ce prix Nobel.

\footnotetext{
${ }^{8}$ César Milstein et Georges Köhler (Medical Research Council Laboratory of Molecular Biology, Cambridge Royaume-Uni) ont reçu le prix Nobel de Physiologie ou Médecine en 1984 pour leur découverte de la technique d'obtention des anticorps monoclonaux [48].
} 
JLT : pour conclure, toi qui es président du Cancéropôle lle-de-France, est-ce que la messe a été dite pour l'immunothérapie des cancers ou bien vers quoi nous orientons-nous, avec ce paradigme du déplacement du traitement de la cellule cancéreuse vers le traitement du système immunitaire du patient?

WHF : non, je crois que la messe est loin d'être dite, une décennie ne s'est même pas écoulée On commence déjà à voir les limites de ces traitements, avec les résistances primaires et, surtout, les résistances secondaires qui se mettent en place, qui nous disent qu'il va falloir trouver d'autres choses. On est véritablement au tout début de l'histoire, le changement de paradigme fait que, effectivement, on va chercher à moduler différemment le système immunitaire de façon encore plus efficace. Toutes les approches qui associent à une reconnaissance de l'antigène sur une cellule tumorale un recrutement de la réaction immunitaire au site tumoral comme les anticorps bispécifiques, mais aussi des anticorps fusionnés à une cytokine, à un facteur de croissance de cellules dendritiques, le granulocyte-macrophage-colony stimulating factor (GM-CSF) par exemple, toutes ces approches visent à élaborer JLT : des molécules qui n'oublient ni la cellule cancéreuse ni le système immunitaire

WHF : oui, je le disais tout à l'heure, le microenvironnement tumoral, c'est un organe immunitaire, moduler cet environnement devient essentiel, grâce à des molécules « intelligentes », comme des récepteurs $T$ solubles qui ouvrent la voie au ciblage des néo-antigènes que les anticorps ont beaucoup plus de mal à cibler, ou des CAR T avec des récepteurs $T$ également Cela, c'est le premier point. Le deuxième point est que tout ce qui a été fait jusqu'à présent a visé essentiellement le lymphocyte; or celui-ci n'est pas la cellule majoritaire dans le microenvironnement tumoral, loin s'en faut ; les cellules myéloïdes, les fibroblastes remodelés, les vaisseaux, également modifiés C'est tout cela qui fait le stroma tumoral, bien plus que les lymphocytes. Nous savons désormais que l'interaction entre ces cellules myéloïdes, fibroblastiques, etc. avec les lymphocytes T mémoire mais aussi les lymphocytes $B$, des cellules à la fois présentatrices d'antigènes et productrices d'anticorps, est indispensable pour contrôler la tumeur à long terme. Troisième point, encore balbutiant, c'est ce qui a fait du système immunitaire le système qui a révolutionné la médecine, la vaccination. Aujourd'hui, lorsqu'il s'agit d'un cancer viro-induit comme le cancer du col de l'utérus ou les hépatocarcinomes, on sait faire cette vaccination antivirale préventive, mais quand on en vient à une vaccination thérapeutique, on bute contre des difficultés de deux ordres: premièrement, on ne sait pas vacciner efficacement contre autre chose qu'un micro-organisme, virus, bactéries, et, deuxièmement, quand on vaccine contre un antigène associé à la tumeur, s'il n'est pas indispensable à la cellule tumorale, il disparaît, une situation un peu semblable à celle de la mise au point de vaccins contre des virus mutants ! Le dernier point, c'est qu'il est désormais clair que toute thérapeutique efficace du cancer est une immunothérapie ! La chimiothérapie, les thérapies ciblées, la radiothérapie, ont donc un nouvel avenir grâce à l'immunothérapie. Tout ce qui augmente l'immunogénicité, les virus oncolytiques par exemple, tous ces éléments qui font que cet organe immunitaire qu'est le microenvironnement tumoral se retrouve modifié par une intervention locale ou à distance, a un avenir potentiel. Je ne sais pas s'il y aura un autre prix Nobel sur les thérapies cellulaires - il y en a déjà eu deux pour les greffes de moelle et de rein - mais, surtout, je pense que le chemin des découvertes pour faire de ce changement de paradigme, qui a moins de dix ans, la voie royale, débute à peine. $\diamond$

From the time in the wilderness to the Nobel Prize in Physiology or Medicine awarded to James Allison and Tasuku Honjo: the Long March of cancer immunotherapy

\section{LIENS D'INTÉRÊT}

Les auteurs déclarent n'avoir aucun lien d'intérêt concernant les données publiées dans cet article.

\section{RÉFÉRENCES}

1. Coley WB. The treatment of malignant tumors by repeated inoculations of erysipelas : with a report of ten original cases. Am J Med Sci 1893; 105 : 487-511.

2. Carswell EA, Old LJ, Kassel RL, Green S, Fiore N, Williamson B. An endotoxininduced serum factor that causes necrosis of tumor. N Engl J Med $1975 ; 72$ : 3666-70.

3. Old LJ, Clark DA, Benacerraf B. Effect of Bacillus Calmette Guérin infection on transplanted tumors in the mouse. Nature 1959 ; $184: 291-2$.

4. Burnet M. Cancer-a biological approach. I. The processes of control. Br Med J $1957 ; 5022: 779-86$.

5. Traversari K, van der bruggen $P$, Luescher IF, et al. A nonapeptide encoded by human Gene MAGE-l is recognized on HLA-Al by cytolytic T lymphocytes directed against tumor antigen MZ2- $\varepsilon$. J Exp Med 1992 ; 176 : 1453-7.

6. Dun GP, Bruce AT, Ikeda H, Old LJ, Schreiber RD. Cancer immunoediting : from immunosurveillance to tumor escape. Nat Immunol $2002 ; 3$ : 991-8.

7. Galon J, Costes A, Sanchez-Cabo F, et al. Type, density, and location of immune cells within human colorectal tumors predict clinical outcome. Science 2006 ; 313 : 1960-4

8. Dieu-Nosjean MC, Antoine M, Danel C, et al. Long-term survival for patients with non-small-cell lung cancer with intratumoral lymphoid structures.J Clin Oncol $2008 ; 26: 4410-7$.

9. Bessis M, Bernard J. Remarquables résultats du traitement par l'exsanguinotransfusion d'un cas de leucémie aiguë. Bull Mem Soc Med Hop Paris 1947 ; $63: 871-7$.

10. Mathé G, Bernard J, Schwarzenberg L, et al. Trial treatment of patients afflicted with acute leukemia in remission with total irradiation followed by homologous bone marrow transfusion. Rev Fr Etud Clin Biol 1959 ; 4 : 675 704

11. Rosenberg SA, Mulé JJ, Spiess PJ, et al. Regression of established pulmonary metastases and subcutaneous tumor mediated by the systemic administration of high-dose recombinant interleukin 2.J Exp Med 1985 ; $161: 1169-88$.

12. Rosenberg SA, Lotze MT, Muul LM, et al. Observations on the systemic administration of autologous lymphokine-activated killer cells and recombinant interleukin-2 to patients with metastatic cancer. $N$ Engl J Med $1985 ; 313: 1485-92$

13. Spiess PJ, Yang JC, Rosenberg SA. In vivo antitumor activity of tumorinfiltrating lymphocytes expanded in recombinant interleukin-2. J Natl Cancer Inst $1987 ; 79$ : 1067-75.

14. Hodi FS, O'Day SJ, McDermott DF, et al. Improved survival with ipilimumab in patients with metastatic melanoma. N Engl J Med 2010 ; 363 : 711-23.

15. Brahmer JR, Drake CG, Wollner I, et al. Phase I study of single-agent antiprogrammed death-1 (MDX-1106) in refractory solid tumors : safety, clinical activity, pharmacodynamics, and immunology correlates. J Clin Oncol $2010 ; 28$ : 3167-75.

16. Gross G, Waks T, Eshhar Z. Expression of immunoglobulin-T-cell receptor chimeric molecules as functional receptors with antibody-type specificity. Proc Natl Acad Sci USA 1989 ; 86 : 10024-8. 


\section{RÉFÉRENCES}

17. Kalos M, Levine BL, Porter DL, et al. T cells with chimeric antigen receptors have potent antitumor effects and can establish memory in patients with advanced leukemia. Sci Transl Med 2011 ; 3 : 95ra73.

18. Brentjens RJ, Davila ML, Rivière I, et al. CD19-targeted T cells rapidly induce molecular remissions in adults with chemotherapy-refractory acute lymphoblastic leukemia. Sci Transl Med 2013 ; 5 :177ra38.

19. Dossett ML, Teague RM, Schmitt TM, et al. Adoptive immunotherapy of disseminated leukemia with TCR-transduced, CD $8{ }^{+} \mathrm{T}$ cells expressing a known endogenous TCR. Mol Ther $2009 ; 17: 742-9$.

20. Miller RA, Maloney DG, Warnke R, Levy R. Treatment of B-cell lymphoma with monoclonal antiidiotype antibody. $N$ Engl J Med 1982 ; 306 : 517-22.

21. Park S, Jiang Z, Mortenson $E D$, et al. The therapeutic effect of anti-HER2/ neu antibody depends on both innate and adaptive immunity. Cancer Cell $2010 ; 18: 160-70$.

22. Abes R, Gélizé $\varepsilon$, Fridman WH, Teillaud JL. Long-lasting anti-tumor protection by anti-CD20 antibody through cellular immune response. Blood $2010 ; 116: 926-34$.

23. Casarès $\mathrm{N}$, Pequignot $M 0$, Tesnière $A$, et al. Caspase-dependent immunogenicity of doxorubicin-induced tumor cell death. J Exp Med 2005 ; 202 : 1691-701

24. Brack C, Hirama M, Lenhard-Schuller, Tonegawa S. A complete immunoglobulin gene is created by somatic recombination. Cell, $1978 ; 15: 1-14$.

25. Breathnach R, Mandel JL, Chambon P. Ovalbumin gene is split in chicken DNA. Nature 1977 ; 270 : 314-9.

26. Tilghman SM, Tiemeier DC, Seidman JG, et al. Intervening sequence of DNA identified in the structural portion of a mouse b-globin gene. Proc Natl Acad Sci USA $1978 ; 75: 725-9$.

27. Honjo T, Kataoka T. Organization of immunoglobulin heavy chain genes and allelic deletion model. Proc Natl Acad Sci USA 1978 ; 75 : 2140-4.

28. Kataoka T, Kawakami T, Takahashi N, Honjo T. Rearrangement of immunoglobulin gl-chain gene and mechanism for heavy-chain class switch. Proc Natl Acad Sci USA $1980 ; 77$ : 919-23.

29. Shimizu A, Akahashi N, Yaoita Y, Honjo T. Organization of the constantregion gene family of the mouse immunoglobulin heavy chain. Cell $1982 ; 28$ : 499-506.

30. Muramatsu M, Sankaranand VS, Anant S, et al. Specific expression of Activation-induced Cytidine Deaminase (AID), a novel member of the RNA-editing deaminase family in germinal center B cells. J Biol Chem 1999 ; $274: 18470-6$.
31. Revy P, Muto T, Levy Y, et al. Activation-induced cytidine deaminase (AID) deficiency causes the autosomal recessive form of the hyper-IgM syndrome (HIMG2). Cell, $2000 ; 102: 565-75$.

32. Muramatsu M, Kinoshita K, Fagarasan S, et al. Class switch recombination and hypermutation require activation-induced cytidine deaminase (AID), a potential RNA editing enzyme. Cell 2000 ; $102: 553-63$

33. Ishida Y, Agata Y, Shibahara K, Honjo T. Induced expression of PD-1, a novel member of the immunoglobulin gene superfamily, upon programmed cell death. EMBO J $1992 ; 11: 3887-95$.

34. Nishimura Y, Nose M, Hiai H, et al. Development of lupus-like autoimmune diseases by disruption of the PD-1 gene encoding an ITIM motif-carrying immunoreceptor. Immunity $1999 ; 11: 141-51$.

35. Okazaki T, Tanaka Y, Nishio R, et al. Autoantibodies against cardiac troponin I are responsible for dilated cardiomyopathy in PD-1-deficient mice. Nat Med $2003 ; 9$ : 1477-83.

36. Freeman GJ, Long AJ, Iwai Y, et al. Engagement of the PD-1 immunoinhibitory receptor by a novel B7 family member leads to negative regulation of lymphocyte activation. J Exp Med 2000; 192 : $1027-34$.

37. Okazaki T, Maeda A, Nishimura H, et al. PD-1 immunoreceptor inhibits B cell receptormediated signaling by recruiting src homology 2-domain-containing tyrosine phosphatase 2 to phosphotyrosine. Proc Natl Acad Sci USA 2001 ; 98 : 13866-71.

38. Amigorena S, Bonnerot C, Drake JR, et al. Cytoplasmic domain heterogeneity and functions of IgG Fc receptors in B lymphocytes. Science $1992 ; 256: 1808-12$.

39. Iwai $Y$, Ishida $Y$, Tanaka $Y$, et al. Involvement of PD-Ll on tumor cells in the escape from host immune system and tumor immunotherapy by PD-L1 blockade. Proc Natl Acad Sci USA 2002 ; 99 : 12293-7.

40. Iwai Y, Terawaki S, Honjo T. PD-1 blockade inhibits hematogenous spread of poorly immunogenic tumor cells by enhanced recruitment of effector T cells. Int Immunol 2005 ; 17 : 133-44.

41. Krummel MF, Allison JP. CD28 and CTLA-4 have opposing effects on the response of T cells to stimulation.J Exp Med 1995; $182:$ 459-65.

42. Walunas TL, Lenschow DJ, Bakker CY, et al. CTLA-4 can function as a negative regulator of T cell activation. Immunity $1994 ; 1: 405-13$.

43. Brunet JF, Denizot F, Luciani MF, et al. A new member of the immunoglobulin superfamily-CTLA-4. Nature $1987 ; 328: 267-70$.

44. Leach DR, Krummel MF, Allison JP. Enhancement of antitumor immunity by CTLA-4 blockade. Science $1996 ; 271: 1734-6$.

45. Kwon ED., Hurwitz AA, Foster BA, et al. Manipulation of T cell costimulatory and inhibitory signals for immunotherapy of prostate cancer. Proc Natl Acad Sci USA 1997 ; $94: 8099-103$.

46. Coley WB. Late results of the treatment of inoperable sarcoma by the mixed toxins of Erysipelas and Bacillus prodigosus. Am J Med Sci $1906 ; 131: 375-430$.

47. Hanahan D, Weinberg RA. Hallmarks of cancer : the next generation. Cell 2011 ; $144: 646-74$.

48. Köhler $\mathrm{G}$, Milstein C. Continuous cultures of fused cells secreting antibody of predefined specificity. Nature $1975 ; 256: 495-7$.

\section{TIRÉS À PART}

\section{J.L. Teillaud}

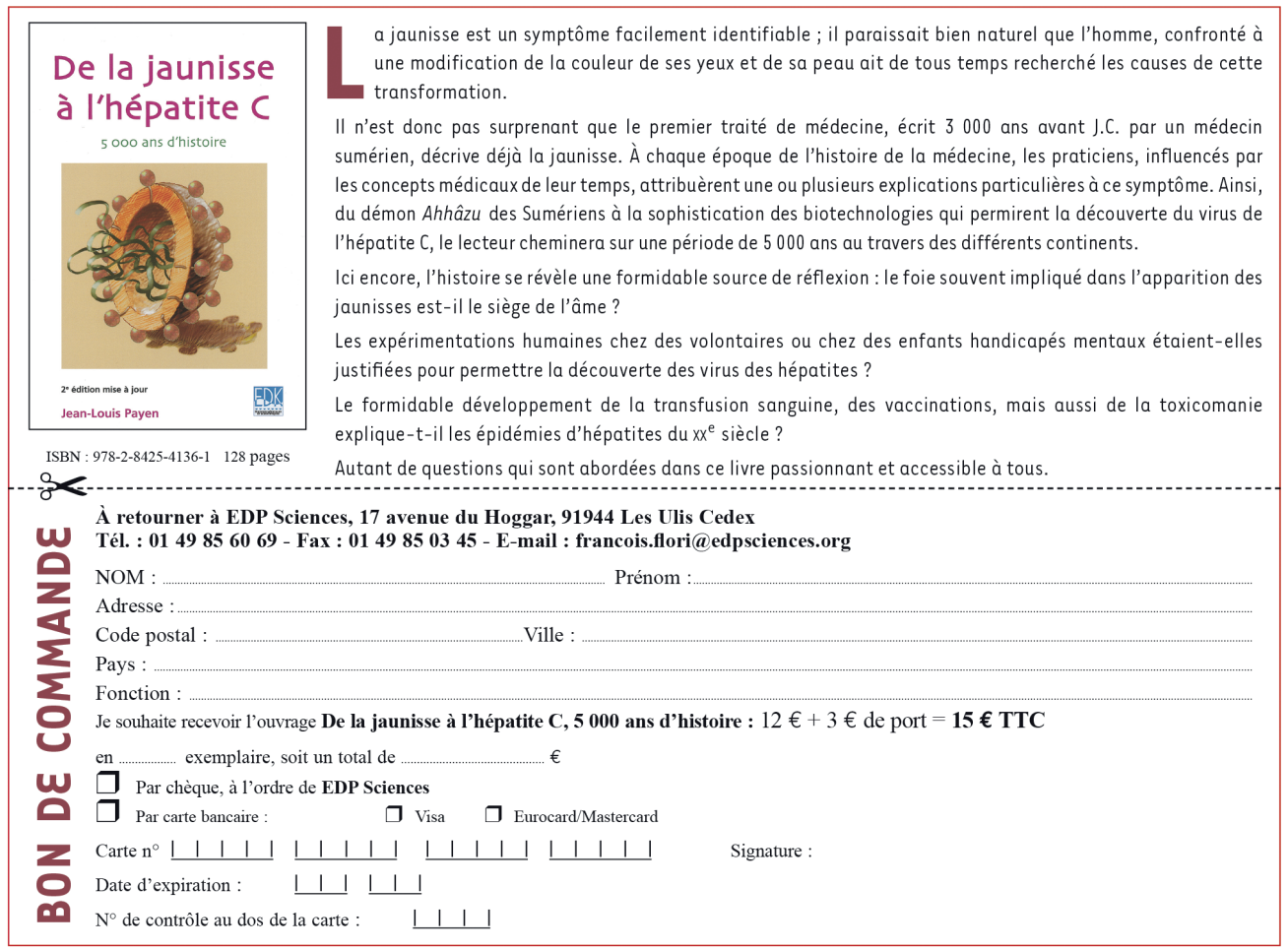

